\title{
Online Learning Mechanisms for Bayesian Models of Word Segmentation
}

\author{
Lisa Pearl · Sharon Goldwater • Mark Steyvers
}

Published online: 18 March 2011

C The Author(s) 2011. This article is published with open access at Springerlink.com

\begin{abstract}
In recent years, Bayesian models have become increasingly popular as a way of understanding human cognition. Ideal learner Bayesian models assume that cognition can be usefully understood as optimal behavior under uncertainty, a hypothesis that has been supported by a number of modeling studies across various domains (e.g., Griffiths and Tenenbaum, Cognitive Psychology, 51, 354-384, 2005; Xu and Tenenbaum, Psychological Review, 114, 245-272, 2007). The models in these studies aim to explain why humans behave as they do given the task and data they encounter, but typically avoid some questions addressed by more traditional psychological models, such as how the observed behavior is produced given constraints on memory and processing. Here, we use the task of word segmentation as a case study for investigating these questions within a Bayesian framework. We consider some limitations of the infant learner, and develop several online learning algorithms that take these limitations into account. Each algorithm can be viewed as a different method of approximating the same ideal learner. When tested on corpora of English child-directed speech, we find that the constrained learner's behavior depends non-trivially on how the learner's limitations are implemented. Interestingly, sometimes biases that are helpful to an ideal learner hinder a constrained learner, and in a few cases, constrained learners perform equivalently or better than the ideal learner. This suggests that the transition from a computational-level solution for acquisition to an algorithmic-level one is not straightforward.
\end{abstract}

\footnotetext{
L. Pearl $(\varangle) \cdot$ M. Steyvers

Department of Cognitive Sciences, University of California, 3151 Social Science Plaza, Irvine,

CA 92697-5100, USA

e-mail: lpearl@uci.edu

S. Goldwater

School of Informatics, University of Edinburgh, Edinburgh, UK
} 
Keywords Algorithmic level · Bayesian models · Computational level · English · Ideal learning · Online learning · Processing limitations · Word segmentation

\section{Introduction}

Language acquisition can be thought of as an induction problem, where the child observes some finite set of linguistic data, and must generalize beyond those data to form a more abstract representation of the language that can be used to produce and understand novel forms. In recent years, there has been growing interest and success in examining induction problems in many areas of cognition using a rational analysis approach (Oaksford and Chater 1998), often through the use of Bayesian models (Griffiths et al. 2008, 2010; Tenenbaum et al. 2006). These models are typically used to examine problems at Marr's (1982) computational level of analysis, asking what the goal of the computation is and the general strategy by which it might be solved. They are ideal learners, which solve the induction problem optimally given particular assumptions about internal representation and the information available to the learner. Researchers have found that human behavior accords with that of the models in a number of domains, including language (e.g., Frank et al. 2009; Griffiths and Tenenbaum 2005; Tenenbaum and Griffiths 2001; Xu and Tenenbaum 2007). These results are useful for showing that humans can integrate data optimally in many cases, but due to their focus on the computational level of explanation, the models tell us very little about the actual processes and mechanisms by which humans might achieve these behaviors - what Marr (1982) termed the algorithmic level of analysis. Indeed, one of the main criticisms of the Bayesian approach is that its frequent neglect of algorithmic-level explanations is unsatisfying to those who are interested in the processes by which humans make their inductive leaps (McClelland et al. 2010). Given the limitations on human cognitive abilities (e.g., memory and processing), what kinds of algorithms might actually be used to compute the solutions that are optimal under a Bayesian model? What kinds of approximations might be required, and will different approximations lead to different results?

In this paper, we begin to address these very general questions by using the task of word segmentation by human infants as a case study. Our work can be viewed as one instance of a recent trend towards examining cognitively plausible implementations of Bayesian models (Brown and Steyvers 2009; Sanborn et al. in press; Shi et al. in press). The problem of word segmentation is useful for our purposes because previous work has already analyzed the behaviors of ideal learners on this task, characterizing the differences between learners making different assumptions about the nature of the input data. Specifically (as discussed further in Sect. 2.2), ideal learners who assume that words are predictive of other words (implemented using a bigram model) are more successful than learners who make the simpler unigram assumption that words are independent units (Goldwater et al. 2009).

The results of Goldwater et al. (2009) (henceforth GGJ) were obtained by implementing their ideal learners using an algorithm that stored the entire corpus in memory. Here we ask whether more cognitively plausible algorithms, which take into account human memory and processing limitations, yield similar results (either quantitatively 
or qualitatively). We develop three different algorithms, where each algorithm can be viewed as a different method of approximating the same ideal learner. Like GGJ's ideal learners, our learners are unsupervised: their input consists of strings of phonemes with no word boundaries marked (except for utterance boundaries), and no initial lexicon of known words. Unlike GGJ's ideal learners, our learners use online (incremental) processing algorithms, which assume that the learner can only store and process a limited amount of data at once. When tested on a corpus of English childdirected speech (Bernstein-Ratner 1984), we find that the modeled learner's behavior depends non-trivially on how the learner's processing limitations are implemented. In particular, not all of the constrained learners exhibit the same qualitative differences between unigram and bigram versions as do the ideal learners. In some cases, the constrained unigram learners actually perform better than the ideal unigram learners, a behavior we discuss in light of Newport's "Less is More" hypothesis about human language acquisition (Newport 1990). These results show that different kinds of cognitive limitations (constraints on the kinds of hypotheses learners entertain-e.g., whether or not words are predictive-and constraints on memory and processing) can interact in surprising and non-trivial ways. In particular, learners with restricted hypothesis spaces (here, unigram learners) may actually benefit from having restricted processing powers as well. Also, although the online learners we explore here are less successful than the most powerful learner we tested (the ideal bigram learner), we find they are able to utilize the statistical information in the data quite well, achieving comparable performance to other recent models of word segmentation, and far better performance than a simple transitional probability learner (Saffran et al. 1996).

\section{Statistical Word Segmentation}

Word segmentation is the task of identifying word boundaries in fluent speech, and is one of the first problems that infants must solve during language acquisition. A number of weak cues to word boundaries are present in fluent speech, and there is evidence that infants are able to use many of these, including phonotactics (Mattys et al. 1999), allophonic variation (Jusczyk et al. 1999b), metrical (stress) patterns (Morgan et al. 1995; Juszcyk et al. 1999c), effects of coarticulation (Johnson and Jusczyk 2001), and statistical regularities among sequences of syllables (Saffran et al. 1996). With the exception of the last cue, all these cues are language-dependent, in the sense that the way the cue relates to word boundaries differs between languages. For example, English words are most often stressed on the initial syllable, while in other languages stress might be more typical on the penultimate or final syllable. Similarly, phonotactic constraints differ between languages, with legal words in one language being illegal in others. Thus, one would normally assume that in order to use these cues, infants must have already learned some of the words in the language in order to identify the dominant stress patterns and phonotactics [though see Blanchard et al. (2010) for one way language-specific phonotactics might be learned at the same time the initial segmentation problem is being solved]. Since the point of word segmentation is to identify words in the first place, needing to know words in order to learn segmentation cues creates a chicken-and-egg problem. Fortunately, language- 
independent cues, such as statistical regularities between syllables or phonemes, can help infants out of this problem by allowing them to identify words (statistically coherent sound sequences) or word boundaries (statistically incoherent sound sequences) without already knowing what some words are. Infants appear to use these regularities earlier than other kinds of cues (Thiessen and Saffran 2003), which suggests that strategies exploiting regularities in syllable or phoneme sequences can indeed provide the initial bootstrapping step for word segmentation. Consequently, although most of the other cues mentioned above are also statistical in nature, research (especially computational research) into statistical word learning has tended to focus on the use of syllable and phoneme regularities.

Before describing the models we will be exploring here, we first briefly review some other unsupervised models of word segmentation. A complete review of previous work is beyond the scope of this paper; instead we describe only some of the most recent models, which we will use for comparison to our own, and refer the reader to Goldwater (2006) for additional references.

\subsection{Recent Statistical Word Segmentation Models}

\subsubsection{WordEnds}

WordEnds (Fleck 2008) is an unsupervised learning algorithm that operates in batch mode over sequences of phonemes. It focuses on boundaries (rather than words), and works by estimating the probabilities of different phoneme sequences occurring at word beginnings and endings, using these to identify locations that are likely to be word boundaries. An initial estimate of the probabilities is made by looking at the sequences that occur at utterance boundaries. These initial probabilities are used to hypothesize new word boundaries, which are then used to update the learner's estimates of probable word beginnings and endings, and the process continues iteratively. As a final cleanup measure, the learner merges hypothesized words together if the merged word occurs frequently enough in the existing segmentation. Although lexical items are used in the last step of the algorithm, WordEnds is not primarily word-based, in that it does not try to optimize a lexicon or explicitly model entire words or their relationships to each other (the phoneme sequences to the right and left of a word boundary are assumed to be independent, so the learner does not assume one word is predictive of the next).

\subsubsection{Bootstrap Voting Experts}

Hewlett and Cohen (2009) introduced a learning model called Bootstrap Voting Experts that works by chunking together sequences of phonemes that have low internal entropy and high boundary entropy. Thus, like WordEnds, this learner uses phonemic probability information, but no explicit model of words or a lexicon. Also like WordEnds, the learner iterates over the corpus in batch mode, improving subsequent segmentation hypotheses by information gained from previous segmentation hypotheses. Using a sliding window of a fixed length, two "voting experts" use accumulated 
entropy knowledge to vote whether a boundary should be inserted between two phonemes; if the number of votes exceeds a pre-determined threshold, the learner inserts a word boundary.

\subsubsection{PHOCUS}

Blanchard et al. (2010) created PHOCUS (PHOnotactic CUe Segmenter), an online learner that couples statistical word learning with phonotactic constraints. In particular, Blanchard et al. demonstrate a way in which language-specific phonotactic constraints, realized as likely and unlikely phonemic sequences, can be learned at the same time that segmentation is being attempted and an explicit lexicon is built. The learner uses an online algorithm, processing one utterance at a time and starting with an initially empty lexicon. The learner considers all possible segmentations of each utterance and chooses the one that is most probable, as computed by multiplying together the probabilities of each hypothesized word. The newly segmented words are then added to the lexicon (or, if they already exist, their counts are incremented). The relative frequency of each word in the lexicon is used as its probability when segmenting future utterances, and phonotactic probabilities are computed based on the current lexical items. Possible words that are not in the lexicon are assigned probabilities using these phonotactic probabilities. Note that when the learner starts out, no lexical items exist, so utterances will not be segmented, but added to the lexicon whole. However, since some utterances are individual words, they allow the learner to begin to find boundaries in later utterances.

This model is the most similar to those we introduce below, in that it uses an online algorithm and computes probabilities of different segmentations based on a learned lexicon. The main differences are in exactly how the probabilities are computed and the use of phonotactic probabilities in the PHOCUS learner. The best-performing variants of this learner also include domain-specific universally applicable knowledge about words - namely, well-formed words must have a least one syllabic sound, and the learner knows which sounds are syllabic.

In addition, some of our learners assume that words are predictive of each other, whereas PHOCUS does not.

\subsection{Bayesian Word Segmentation}

The starting point of our research is the work of Goldwater et al. (2009) (GGJ), which provides a Bayesian learning analysis of how statistical information could be used by infants to begin to segment words from continuous speech. It is a computational-level approach which defines the goal of learning as identifying the optimal segmentation of the input corpus from the space of all possible segmentations. Each segmentation implicitly defines a lexicon (the set of word types occurring in the segmentation); the learner decides which segmentation is optimal based on the words in the learned lexicon and their frequencies.

In the language of Bayesian analysis, the learner seeks to identify an explanatory hypothesis that both accounts for the observed data and conforms to prior expectations 
about what a reasonable hypothesis should look like. GGJ develop two models within a Bayesian framework where the learner is presented with some data $d$ (a corpus of phonemically transcribed utterances, where each utterance is an unsegmented sequence of phonemes) ${ }^{1}$ and seeks a hypothesis $h$ (a segmentation of the corpus into a sequence of words) that both explains the data (i.e., concatenating together the words in $h$ forms $d$ ) and has high prior probability. The optimal solution is the hypothesis with the highest probability, given the data:

$$
P(h \mid d) \propto P(d \mid h) P(h)
$$

The learner determines the posterior probability of $h$ having observed $d$ based on $P(d \mid h)$ - the likelihood of $d$ being observed if $h$ was true-and $P(h)$ - the prior probability of $h$. Since a hypothesis is only a sequence of words, if the hypothesis sequence matches the observed sequence of phonemes, the likelihood is 1 ; if the hypothesis sequence does not match the observed sequence, the likelihood is 0 . For example, hypotheses consistent with the observation sequence lookatthedoggie (we use orthographic rather than phonemic transcriptions here for clarity) include lookatthedoggie, look at the doggie, lo oka th edo ggie, and lookatthedoggie. Inconsistent hypotheses, for which $P(d \mid h)=0$, include $i$ like pizza, a $b c$, and lookatthat.

Since the likelihood is either 0 or 1 , all of the work in the models is done by the prior distribution over hypotheses. For GGJ, the prior of $h$ encodes the intuitions that words should be relatively short, and the lexicon should be relatively small. In addition, each of the two models encodes a different expectation about word behavior: in the unigram model, the learner assumes that words are statistically independent (i.e. context is not predictive); in the bigram model, words are assumed to be predictive units.

To encode these intuitions mathematically, GGJ use a model based on the Dirichlet Process from nonparametric Bayesian statistics (Ferguson 1973), which can be summarized as follows. Imagine that the sequence of words $w_{1} \ldots w_{n}$ in $h$ is generated sequentially using a probabilistic generative process. In the unigram model, the identity of the $i$ th word is chosen according to

$$
P\left(w_{i}=w \mid w_{1} \ldots w_{i-1}\right)=\frac{n_{i-1}(w)+\alpha P_{0}(w)}{i-1+\alpha}
$$

where $n_{i-1}(w)$ is the number of times $w$ has occurred in the previous $i-1$ words, $\alpha$ is a parameter of the model, and $P_{0}$ is a base distribution specifying the probability that a novel word will consist of the phonemes $x_{1} \ldots x_{m}$ :

$$
P_{0}\left(w=x_{1} \ldots x_{m}\right)=\prod_{j=1}^{m} P\left(x_{j}\right)
$$

\footnotetext{
1 Note that the units over which these models operate are phonemes, rather than phonetic features (Christiansen et al. 1998) or syllables (Swingley 2005). This is not uncontroversial, as it makes the model insensitive to feature-based similarity between sounds and abstracts away from many details of phonetic and acoustic variation. However, it was chosen based on the available input corpora, and to facilitate comparison with other word segmentation models.
} 
The equation in (2) enforces the preference for a small lexicon by stating that the probability of a word is approximately proportional to the number of times that word has occurred previously. Thus, hypotheses where a small number of words occur frequently will be preferred over those with larger lexicons, where each word occurs less often. In addition, the first time a word appears in the sequence, $n_{i-1}(w)=0$, so the probability of the word is completely determined by the equation in (3). Since (3) is a product of the phonemes in the word, words with fewer phonemes (i.e., shorter words) will be preferred. The GGJ model also includes a geometric distribution over utterance lengths, to account for the fact that the corpus consists of individual utterances. A more detailed description of both the unigram model and the bigram model (below) can be found in the original paper.

The bigram model, which is based on a hierarchical Dirichlet Process (Teh et al. 2006), is conceptually similar to the unigram model except that it tracks not only the frequencies of individual words, but also the frequencies of pairs of words. Just as the unigram model prefers hypotheses where a small number of words appear with high frequency, the bigram model prefers hypotheses where a small number of bigrams appear with high frequency (in addition to the assumptions of the unigram model). The model is defined as follows:

$$
\begin{aligned}
& P\left(w_{i}=w \mid w_{i-1}=w^{\prime}, w_{1} \ldots w_{i-2}\right)=\frac{n_{i-1}\left(w^{\prime}, w\right)+\beta P_{1}(w)}{n_{i-1}\left(w^{\prime}\right)+\beta} \\
& P_{1}\left(w_{i}=w\right)=\frac{b_{i-1}(w)+\gamma P_{0}(w)}{b_{i-1}+\gamma}
\end{aligned}
$$

where $n_{i-1}\left(w^{\prime}, w\right)$ is the number of times the bigram $\left(w^{\prime}, w\right)$ has occurred in the first $i-1$ words, $b_{i-1}(w)$ is the number of times $w$ has occurred as the second word of a bigram, $b_{i-1}$ is the total number of bigrams, and $\beta$ and $\gamma$ are model parameters. The preference for hypotheses with relatively few distinct bigrams is enforced in the equation in (4), by making a bigram's probability approximately proportional to the number of times it has occurred before. This is analogous to the equation in (2) for the unigram model. When a new bigram is created, its probability is determined by the equation in (5), which assigns higher probability to new bigrams that use words that already occur in many other bigrams (i.e., the model assumes that a few words create bigrams very promiscuously, while most do not).

\subsection{Ideal and Constrained Bayesian Inference}

\subsubsection{Ideal Learners}

To evaluate the performance of both the unigram and bigram Bayesian models in an ideal learner framework, GGJ used Gibbs sampling, a stochastic search procedure often used for ideal learner inference problems. Gibbs sampling, a type of Markov chain Monte Carlo procedure, is a batch algorithm that iterates over the corpus multiple times. Gibbs samplers are guaranteed to converge, which means that after a number of initial iterations (usually called "burn-in"), each iteration produces a sample from the 
posterior distribution of the model in question (here, either the unigram or bigram GGJ model). This convergence guarantee is what makes these samplers popular for ideal learner problems, since it means that the true posterior of the model can be examined without the effects of additional constraints imposed by the learning algorithm.

During each iteration of GGJ's Gibbs sampler, every possible boundary location (position between two phonemes) in the corpus is considered in turn. At each location $b$, the probability that $b$ is a boundary is computed, given the current boundary locations in the rest of the corpus (details of this computation can be found in GGJ; critically, it is based on the equations defining the Bayesian model and thus on the lexicon and frequencies implicit in the current segmentation). Then the segmentation is updated by inserting or removing a boundary at $b$ according to this probability, and the learner moves on to the remaining boundary locations. Pseudocode for this algorithm is shown in (6).

Pseudocode for Gibbs sampler (Ideal Learner)

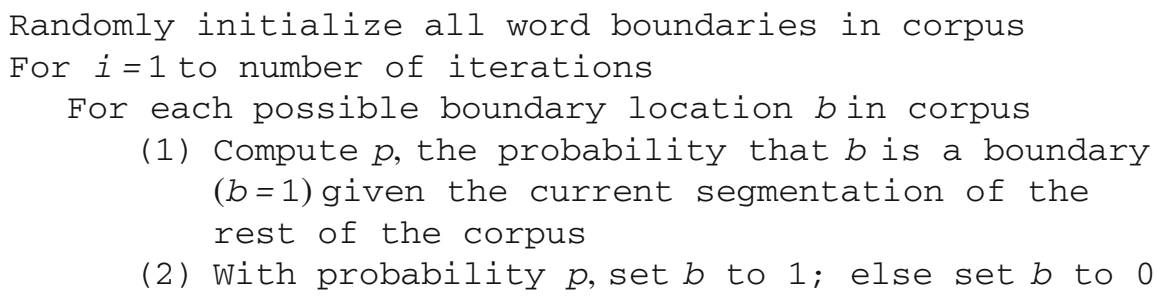

GGJ found that in order to converge to a good approximation of the posterior, the Gibbs sampler required 20000 iterations (i.e., each possible boundary in the corpus was sampled 20000 times), with $\alpha=20$ for the unigram models, and $\beta=10, \gamma=3000$ for the bigram models.

Due to the convergence guarantees noted above, this algorithm is well-suited to the computational-level analysis that GGJ were interested in, allowing them to ask what kinds of segmentations would be learned by ideal learners with different assumptions about the nature of language. GGJ discovered that an ideal learner that is biased to heed context (the bigram model) achieves far more successful segmentation than one that is not (the unigram model). Moreover, a unigram ideal learner will severely undersegment the corpus, identifying common collocations as single words (e.g., you want segmented as youwant), most likely because the only way a unigram learner can capture strong word-to-word dependencies is to assume those words are actually a single word. This tells us about the expected behavior in learners who are able to make optimal use of their input - that is, what in principle are the useful biases for humans to use, given the available data.

Turning to the algorithmic level of analysis, however, the GGJ learner is clearly less satisfactory, since the Gibbs sampling algorithm requires the learner to store the entire corpus in memory, and also to perform a significant amount of processing (recall that each boundary in the corpus is sampled 20000 times). In the following section, we describe three algorithms that make more cognitively plausible assumptions about memory and processing. These algorithms will allow us to investigate how such 
memory and processing limitations might affect the learner's ability to achieve the optimal solution to the segmentation task (i.e., the solution found by the ideal learners in GGJ).

\subsubsection{Constrained Learners}

To simulate limited resources, all the learning algorithms we present operate in an online fashion, so that processing occurs one utterance at a time rather than over the entire corpus simultaneously. Under GGJ's Bayesian model, the only information necessary to compute the probability of any particular segmentation of an utterance is the number of times each word (or bigram, in the case of the bigram model) has occurred in the model's current estimation of the segmentation. Thus, in each of our online learners, the lexicon counts are updated after processing each utterance (and in the case of one learner, during the processing of each utterance as well). The primary differences between our algorithms lie in the additional details of how resource limitations are implemented, and whether the learner is assumed to sample segmentations from the posterior distribution or choose the most probable segmentation. $^{2}$

\subsubsection{Dynamic Programming Maximization}

We first tried to find the most direct translation of the ideal learner to an online learner that must process utterances one at a time, such that the only limitation is that utterances must be processed one at a time. One idea for this is an algorithm we call Dynamic Programming Maximization (DPM), which processes each utterance as a whole, using dynamic programming (specifically the Viterbi algorithm) to efficiently compute the highest-probability segmentation of that utterance given the current lexicon. ${ }^{3}$ It then adds the words from that segmentation to the lexicon and moves to the next utterance. This algorithm is the only one of our three that has been previously applied to word segmentation (Brent 1999). Pseudocode for this learner is shown in (7).

\footnotetext{
${ }^{2}$ We note also that some of the algorithms differ on whether they would converge on the optimal solution, given infinite resources (infinite iterations, infinite memory buffers, etc.). The first algorithm (Dynamic Programming Maximization) would not, while the other two (Dynamic Programming Sampling, Decayed Markov Chain Monte Carlo) would. In this sense, the latter two algorithms might be considered ideal, though the particular implementations here (with their limited resources) are not. Under this view, we are comparing both a constrained non-ideal algorithm and constrained ideal algorithms against an unconstrained ideal algorithm. While all ideal algorithms may be asymptotically equivalent, we will find they exhibit interesting differences given finite resources, and that not all of them compare favorably with the constrained non-ideal algorithm.

3 Technically, the probabilities computed by the Viterbi algorithm (and the forward algorithm used by the DPS model) are only an approximation of the true probabilities of the segmented utterances, since they are based on the contents of the lexicon not including any of the words in the current utterance. Equation (2) shows that in the true posterior, the words segmented at the beginning of the utterance will affect the probabilities of the words at the end of the utterance.
} 


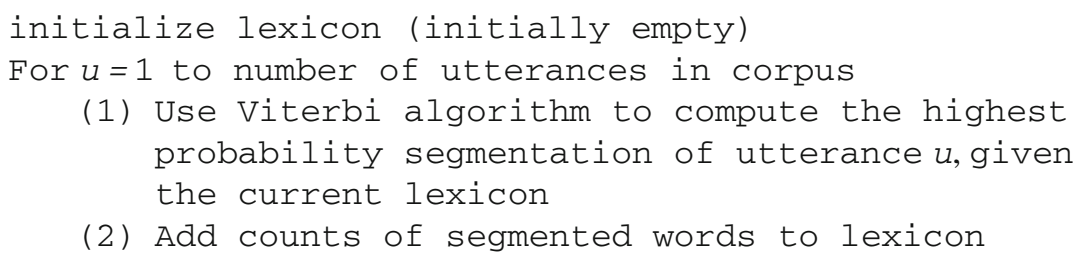

\subsubsection{Dynamic Programming Sampling}

We then created a variant that is similar to DPM, but instead of choosing the most probable segmentation of each utterance conditioned on the current lexicon, it chooses a segmentation based on how probable that segmentation is. This algorithm, called Dynamic Programming Sampling (DPS), computes the probabilities of all possible segmentations using the forward pass of the forward-backward algorithm, and then uses a backward pass to sample from the distribution over segmentations. Pseudocode for this learner is shown in (8); the backward sampling pass is an application of the general method described in Johnson et al. (2007).

Pseudocode for DPS learner

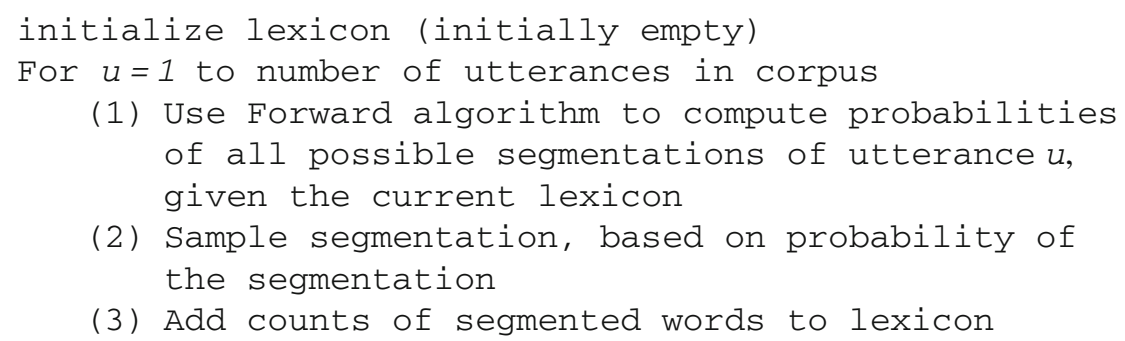

\subsubsection{Decayed Markov Chain Monte Carlo}

We also examined a learning algorithm that recognizes that human memory decays over time and so focuses processing resources more on recent data than on data heard further in the past (a recency effect). We implemented this using a Decayed Markov Chain Monte Carlo (DMCMC) algorithm (Marthi et al. 2002), which processes an utterance by probabilistically sampling $s$ word boundaries from all the utterances encountered so far. The sampling process is similar to Gibbs sampling, except that the learner only has the information available from the utterances encountered so far to inform its decision, rather than information derived from processing the entire corpus.

The probability that a particular potential boundary $b$ is sampled is given by the exponentially decaying function $b_{a}^{-d}$, where $b_{a}$ is the number of potential boundary locations between $b$ and the end of the current utterance, and $d$ is the decay rate. Thus, the further $b$ is from the end of the current utterance, the less likely it is to be sampled. The exact probability is based on the decay rate $d$. For example, suppose $d$ was 1 , and 
Table 1 Likelihood of sampling a given boundary in DMCMC, $d=1$

\begin{tabular}{lll}
\hline Boundary position & $b_{a}^{-d}$ & Relative probability \\
\hline End-1 & $1^{-1}=1 / 1=1.00$ & $1.00 /(\Sigma($ probs $))=0.44$ \\
End-2 & $2^{-1}=1 / 2=0.50$ & $0.50 /(\Sigma($ probs $))=0.22$ \\
End-3 & $3^{-1}=1 / 3=0.33$ & $0.33 /(\Sigma($ probs $))=0.15$ \\
End-4 & $4^{-1}=1 / 4=0.25$ & $0.25 /(\Sigma($ probs $))=0.11$ \\
End-5 & $5^{-1}=1 / 5=0.20$ & $0.20 /(\Sigma($ probs $))=0.08$ \\
\hline
\end{tabular}

The relative probability of a given boundary being sampled is the decay probability $b_{a}^{-d}$ divided by the sum of the decay probabilities for all boundary positions under consideration (in this example, five boundary positions)

there are 5 potential boundaries that have been encountered so far. The probabilities for sampling each boundary are shown in Table 1 .

After each boundary sample is completed, the learner updates the lexicon. Pseudocode for this learner is shown in (9).

\section{Pseudocode for DMCMC learner}

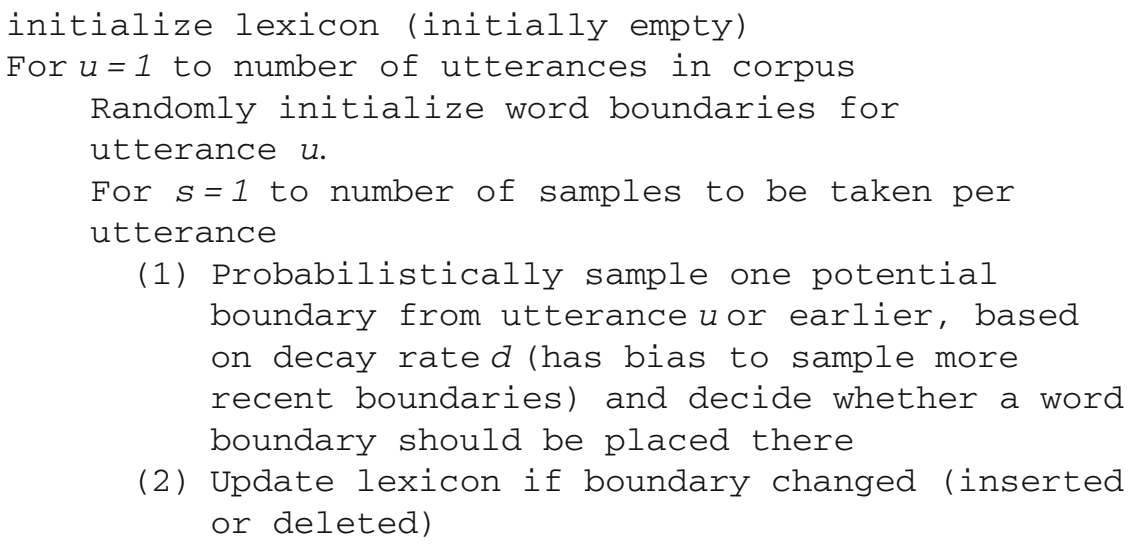

(1) Probabilistically sample one potential boundary from utterance u or earlier, based on decay rated (has bias to sample more recent boundaries) and decide whether a word boundary should be placed there

(2) Update lexicon if boundary changed (inserted or deleted)

We note that one main difference between the DMCMC learner and the Ideal learner is that the Ideal learner samples every boundary from the corpus on each iteration, rather than being restricted to a certain number from the current utterance or earlier. The Ideal learner thus has knowledge of future utterances when making its decisions about the current utterance and/or previous utterances, while the DMCMC learner does not. ${ }^{4}$ In addition, restricting the number of samples in the DMCMC learner means that it requires less processing time/resources than the Ideal learner.

\footnotetext{
${ }^{4}$ We note, however, that the DMCMC learner does have knowledge of "future" utterances when it samples boundaries from utterances further back in the corpus than the current utterance. However, this knowledge of the "future" utterances (compared to the utterance being sampled) only extends to the current utterance, rather than to the whole corpus (which includes utterances after the current utterance). Only at the very end of the corpus would a DMCMC learner have knowledge of the entire corpus when doing any of its samples—but it does not have this knowledge initially, while the Ideal learner does.
} 
Table 2 Probability of sampling a boundary from the current utterance, based on decay rate

\begin{tabular}{ll}
\hline Decay rate & Probability of sampling within current utterance \\
\hline 2.0 & 0.942 \\
1.5 & 0.772 \\
1.0 & 0.323 \\
0.75 & 0.125 \\
0.50 & 0.036 \\
0.25 & 0.009 \\
0.125 & 0.004 \\
\hline
\end{tabular}

We examined a number of different decay rates, ranging from 2.0 down to 0.125 . To give a sense of what these really mean for the DMCMC learner, Table 2 shows the probability of sampling a boundary within the current utterance assuming the learner could sample a boundary from any utterances that occurred within the last $30 \mathrm{~min}$ of verbal interaction (i.e., this includes child-directed speech as well as any silences or pauses in the input stream). Calculations are based on samples from the alice2.cha file from the Bernstein corpus, where an utterance occurs on average every $3.5 \mathrm{~s}$. As we can see, the lower decay rates cause the learner to look further back in time, and thus require the learners to have a stronger memory in order to successfully complete the boundary decision process.

The DMCMC learner has some similarity to previous work on probabilistic human memory, such as Anderson and Schooler (2000). Specifically, Anderson and Schooler argue for a rational model of human memory that calculates a "need" probability for accessing words, which is approximately how likely humans are to need to retrieve that word. The higher a word's need probability, the more likely a human is to remember it. The need probability is estimated based on statistics of the linguistic environment. Anderson and Schooler demonstrate that the need probability estimated from a number of sources, including child-directed speech, appears to follow a power law distribution with respect to how much time has elapsed since the word was last mentioned. Our DMCMC learner, when doing its constrained inference, effectively calculates a need probability for potential word boundaries - this is the sampling probability calculated for a given boundary, which is derived from an exponential decay function. Potential word boundaries further in the past are less likely to be needed for inference, and so are less likely to be retrieved by our DMCMC learner.

\section{Bayesian Model Results}

\subsection{The Data Set}

We tested the GGJ Ideal learner and our three constrained learners on data from the Bernstein corpus (Bernstein-Ratner 1984) from the CHILDES database (MacWhinney 
Table 3 Samples of Bernstein corpus

\begin{tabular}{ll}
\hline English orthography & Phonemic transcription \\
\hline you want to see the book & yu want tu si D6 bUk \\
look there's a boy with his hat & lUk D*z 6 b7 wIT hIz h\&t \\
and a doggie & \&nd 6 dOgi \\
you want to look at this & yu want tu lUk \&t DIs \\
\hline
\end{tabular}

2000). ${ }^{5}$ We used the phonemic transcription of this corpus that has become standard for testing word segmentation models (Brent 1999). The phonemically transcribed corpus contains 9790 child-directed speech utterances (33399 tokens, 1321 types, average utterance length $=3.4$ words, average word length $=2.9$ phonemes). See Table 3 for sample transcriptions and Appendix Fig. A1 for the phonemic alphabet used. Unlike previous work, we used cross-validation to evaluate our models, splitting the corpus into five randomly generated training sets $(\sim 8800$ utterances each) and separate test sets ( $\sim 900$ utterances each), where each training and test set were non-overlapping subsets of the data set used by GGJ. We used separate training and test sets to examine the modeled learner's ability to generalize to new data it has not seen before (and been iterating over, in the case of the Ideal learner). Specifically, we wanted to test if the lexicon the learner inferred was useful beyond the immediate dataset it trained on. Temporal order of utterances was preserved in the training and test sets, such that utterances in earlier parts of each set appeared before utterances in later parts of each set. $^{6}$

\subsection{Performance Measures}

We assessed the performance of these different learners, based on precision and recall over word tokens, word boundaries, and lexicon items, where precision is (\# correct)/(\# found) and recall is (\# correct)/(\# found in the gold standard). To demonstrate how these measures gauge performance differently, let us consider the evaluation of the utterances "look at the doggie" and "look at the kitty", which are translated into phoneme characters as "IUk \&t D6 dOgi" and "IUk \&t D6 kIti". Suppose the algorithm decided the best segmentation was "IUk\&t D6 dOgi" and "IUk\&t D6kIti". For word tokens, precision is $2 / 5$, while recall is $2 / 8$; for word boundaries (utterance-initial and utterance-final boundaries are excluded), precision is $3 / 3$, while recall is $3 / 6$; for lexicon items, precision is $2 / 4$, while recall is $2 / 5$.

\footnotetext{
${ }^{5}$ We note that the statistical strategies we explore here are meant to be an initial bootstrapping method for children to break into word segmentation —as such, we believe testing these strategies on a corpus this size is not unreasonable. However, see additional results from a larger English corpus in the Discussion section that follow the same trends observed in the Bernstein corpus.

${ }^{6}$ Although the Ideal learner is not sensitive to the order of presentation in the training set, the constrained learners are, since they process the data incrementally and early segmentation decisions impact future segmentation decisions.
} 
Fig. 1 Word token F-scores for each of the learners, averaged over the test sets

\section{Learner performance: word tokens}

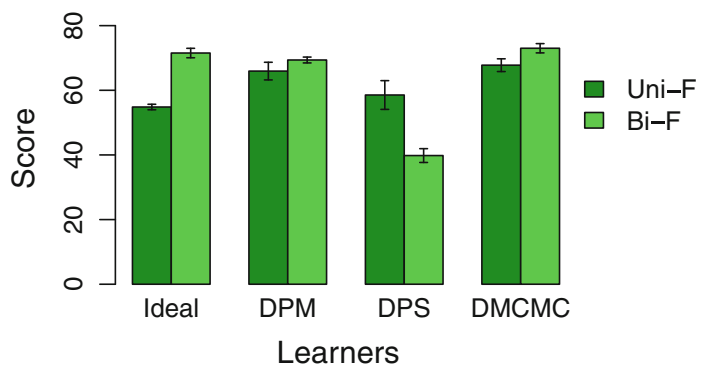

\subsection{Performance}

Table 4 reports the scores for each Bayesian learner, along with results (where available) from other statistical learners discussed previously. $\mathrm{F}$ scores $(\mathrm{F}=(2 *$ precision * recall $) /($ precision + recall)) are also provided. Note that the results for the WordEnds, Bootstrap Voting Experts (BVE), and PHOCUS learners are not strictly comparable to our own results, since they do not use a separate training and test set. Instead, they trained over the entire Bernstein corpus and were evaluated by how well they segmented that corpus. In addition, PHOCUS performance was computed after discarding the first 1000 utterances [see Blanchard et al. (2010) for discussion as to why]. The results for the transitional probability learner are from an implementation that computes transitional probabilities based on an initial sweep through the corpus (technically making it a batch algorithm; online versions are possible but would require some form of smoothing to avoid zero-probability transitions). The learner then inserts boundaries at each transitional probability minimum, as suggested by Saffran et al. (1996). (One could segment instead at a fixed transitional probability threshold; we found no threshold that worked better than using the minimum. ${ }^{7}$

As for the parameters of our own learners, all DMCMC learners have $s=20000$ (20000 samples per utterance), as we found this gave the best segmentation performance. While this may still seem like a lot of processing, this learner nonetheless takes $89 \%$ fewer samples than the ideal learner in GGJ, which is a substantial savings in processing resources. In addition, the DMCMC unigram learners fared best with $d=1.0$, while the DMCMC bigram learners fared best with $d=0.25$. Figure 1 shows the F scores over word tokens for each of the Bayesian learners (both unigram and bigram variants) while Fig. 2 shows the F scores over lexicon items.

A few observations: First, while there is considerable variation in the performance of our constrained learners, all of them out-perform a transitional probability learner operating over phonemes (compare Bayesian learner results to TransProb learner results in Table 4). In addition, our best Bayesian learners compare favorably to other statistical

\footnotetext{
7 We note that our transitional probability learner achieves comparable or better performance compared to other reported transitional probability learner implementations: The one in Brent (1999) operated over phonemes and had token F-scores in the 40s and lexicon precision near 15, while the one in Gambell and Yang (2006) operated over syllables and had token F-scores near 30.
} 
Fig. 2 Lexicon item F-scores for each of the learners, averaged over the test sets

\section{Learner performance: lexicon items}

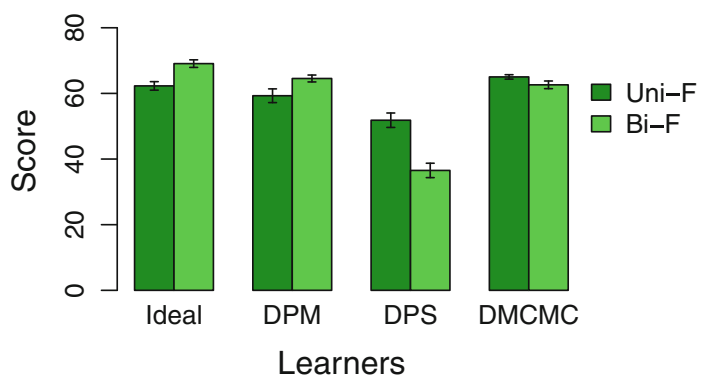

learning algorithms, particularly with respect to their scores over lexicon items. For example, though the batch-learning WordEnds model achieves comparable boundary scores (BF) to many of our online Bayesian learners, its lexicon score (LF) is much lower than most of our online Bayesian learners (LF WordEnds $=36.6$, LF all learners but Bigram DPS: 51.8-65.0). Similarly, while PHOCUS achieves comparable token scores to our best online Bayesian learner (TF PHOCUS: 75.8, TF Bigram DMCMC: 73.0), its lexicon score is lower (LF PHOCUS: 54.5, LF Bigram DMCMC: 62.6). Thus, our online Bayesian learners seem better able to extract a reliable lexicon from the available data than other recent statistical learners, including one (PHOCUS) that relies on domain-specific knowledge about word well-formedness.

Second, when we examine the impact of the unigram and bigram assumptions on word token performance, we find that the bigram learners do not always benefit from assuming words are predictive of other words. While the Ideal, DPM and DMCMC learners do (bigram F > unigram F, Ideal: $p<.001$, DPM: $p=.046$, DMCMC: $p=.002$ ), the DPS learner is harmed by this bias (unigram F > bigram F: $p<.001$ ). This is also true for the lexicon F scores: While the Ideal and DPM learners are helped (bigram F $>$ unigram F, Ideal: $p<.001$, DPM: $p=.002$ ), the DPS and DMCMC learners are harmed (unigram F > bigram F, DPS: $p<.001$, DMCMC: $p=.006$ ) ${ }^{8}$

Third, when comparing our ideal learner to our constrained learners, we findsomewhat unexpectedly - that some of our constrained learners are performing equivalently or better than their ideal counterparts. For example, when we look at word token F-scores for our bigram learners, the DMCMC learner seems to be performing equivalently to the Ideal learner (DMCMC $\neq$ Ideal: $p=0.144$ ). Among the unigram learners, our DPM and DMCMC learners are equally outperforming the Ideal learner (DPM >Ideal: $p<.001$, DMCMC $>$ Ideal: $p<.001$, DPM $\neq$ DMCMC: $p=0.153$ ) and the DPS is performing equivalently to the Ideal learner (Ideal $\neq$ DPS: $p=0.136$ ). Turning to the lexicon F-scores, the results look a bit more expected for the bigram learners: The Ideal learner is out-performing the constrained learners (Ideal $>$ DPM: $p<.001$, Ideal $>$ DPS: $p<$ .001 , Ideal $>$ DMCMC: $p<.001)$. However, among the unigram learners we again

\footnotetext{
8 All $p$-values reported above and below were calculated by comparing 5 runs (1 per test set) of each of the mentioned learners in a two-tailed $t$-test analysis (i.e., 5 observations from each learner were aggregated, and compared against each other).
} 
Table 4 Average performance of different learners on the five test sets, along with published results from other recent statistical learners where available and the results from a transitional probability learner

\begin{tabular}{|c|c|c|c|c|c|c|c|c|c|}
\hline & TP & TR & $\mathrm{TF}$ & BP & BR & $\mathrm{BF}$ & LP & LR & LF \\
\hline \multicolumn{10}{|c|}{ Bayesian Unigram Learners (words are not predictive) } \\
\hline GGJ-Ideal & $\begin{array}{l}63.2 \\
(0.99)\end{array}$ & $\begin{array}{l}48.4 \\
(0.80)\end{array}$ & $\begin{array}{l}54.8 \\
(0.85)\end{array}$ & $\begin{array}{l}92.8 \\
(0.67)\end{array}$ & $\begin{array}{l}62.1 \\
(0.42)\end{array}$ & $\begin{array}{l}74.4 \\
(0.42)\end{array}$ & $\begin{array}{l}54.0 \\
(0.92)\end{array}$ & $\begin{array}{l}73.6 \\
(1.89)\end{array}$ & $\begin{array}{l}62.3 \\
(1.30)\end{array}$ \\
\hline DPM & $\begin{array}{l}63.7 \\
(2.82)\end{array}$ & $\begin{array}{l}68.4 \\
(2.68)\end{array}$ & $\begin{array}{l}65.9 \\
(2.73)\end{array}$ & $\begin{array}{l}77.2 \\
(1.86)\end{array}$ & $\begin{array}{l}85.3 \\
(1.67)\end{array}$ & $\begin{array}{l}81.0 \\
(1.64)\end{array}$ & $\begin{array}{l}61.9 \\
(2.17)\end{array}$ & $\begin{array}{l}56.9 \\
(2.07)\end{array}$ & $\begin{array}{l}59.3 \\
(2.09)\end{array}$ \\
\hline DPS & $\begin{array}{l}55.0 \\
(4.82)\end{array}$ & $\begin{array}{l}62.6 \\
(3.99)\end{array}$ & $\begin{array}{l}58.5 \\
(4.45)\end{array}$ & $\begin{array}{l}70.4 \\
(3.73)\end{array}$ & $\begin{array}{l}84.21 \\
(1.79)\end{array}$ & $\begin{array}{l}76.7 \\
(2.85)\end{array}$ & $\begin{array}{l}54.8 \\
(1.64)\end{array}$ & $\begin{array}{l}49.2 \\
(3.14)\end{array}$ & $\begin{array}{l}51.8 \\
(2.2)\end{array}$ \\
\hline DMCMC & $\begin{array}{l}71.2 \\
(1.57)\end{array}$ & $\begin{array}{l}64.7 \\
(2.31)\end{array}$ & $\begin{array}{l}67.8 \\
(1.97)\end{array}$ & $\begin{array}{l}88.8 \\
(0.89)\end{array}$ & $\begin{array}{l}77.2 \\
(2.17)\end{array}$ & $\begin{array}{l}82.6 \\
(1.53)\end{array}$ & $\begin{array}{l}61.0 \\
(1.18)\end{array}$ & $\begin{array}{l}69.6 \\
(0.43)\end{array}$ & $\begin{array}{l}65.0 \\
(0.67)\end{array}$ \\
\hline \multicolumn{10}{|c|}{ Bayesian Bigram Learners (words are predictive) } \\
\hline GGJ-Ideal & $\begin{array}{l}74.5 \\
(1.41)\end{array}$ & $\begin{array}{l}68.8 \\
(1.53)\end{array}$ & $\begin{array}{l}71.5 \\
(1.46)\end{array}$ & $\begin{array}{l}90.1 \\
(0.75)\end{array}$ & $\begin{array}{l}80.4 \\
(1.01)\end{array}$ & $\begin{array}{l}85.0 \\
(0.82)\end{array}$ & $\begin{array}{l}65.0 \\
(1.19)\end{array}$ & $\begin{array}{l}73.5 \\
(1.71)\end{array}$ & $\begin{array}{l}69.1 \\
(1.15)\end{array}$ \\
\hline DPM & $\begin{array}{l}67.5 \\
(1.13)\end{array}$ & $\begin{array}{l}71.3 \\
(0.74)\end{array}$ & $\begin{array}{l}69.4 \\
(0.90)\end{array}$ & $\begin{array}{l}80.4 \\
(0.96)\end{array}$ & $\begin{array}{l}86.8 \\
(0.63)\end{array}$ & $\begin{array}{l}83.5 \\
(0.57)\end{array}$ & $\begin{array}{l}66.0 \\
(1.00)\end{array}$ & $\begin{array}{l}63.2 \\
(1.46)\end{array}$ & $\begin{array}{l}64.5 \\
(1.05)\end{array}$ \\
\hline DPS & $\begin{array}{l}34.2 \\
(2.16)\end{array}$ & $\begin{array}{l}47.6 \\
(2.16)\end{array}$ & $\begin{array}{l}39.8 \\
(2.13)\end{array}$ & $\begin{array}{l}54.9 \\
(1.40)\end{array}$ & $\begin{array}{l}85.3 \\
(2.07)\end{array}$ & $\begin{array}{l}66.8 \\
(1.00)\end{array}$ & $\begin{array}{l}39.0 \\
(2.02)\end{array}$ & $\begin{array}{l}34.4 \\
(2.42)\end{array}$ & $\begin{array}{l}36.5 \\
(2.19)\end{array}$ \\
\hline DMCMC & $\begin{array}{l}72.0 \\
(1.24)\end{array}$ & $\begin{array}{l}74.0 \\
(1.76)\end{array}$ & $\begin{array}{l}73.0 \\
(1.43)\end{array}$ & $\begin{array}{l}84.1 \\
(0.98)\end{array}$ & $\begin{array}{l}87.4 \\
(1.47)\end{array}$ & $\begin{array}{l}85.7 \\
(0.94)\end{array}$ & $\begin{array}{l}61.1 \\
(1.41)\end{array}$ & $\begin{array}{l}64.2 \\
(1.35)\end{array}$ & $\begin{array}{l}62.6 \\
(1.17)\end{array}$ \\
\hline \multicolumn{10}{|c|}{ Comparison Learners } \\
\hline WordEnds & & & 70.7 & 94.6 & 73.7 & 82.9 & & & 36.6 \\
\hline BVE & 79.1 & 79.4 & 79.3 & 92.8 & 90.5 & 91.6 & & & \\
\hline PHOCUS & 77.7 & 74.0 & 75.8 & 89.7 & 83.6 & 86.5 & 47.3 & 64.0 & 54.5 \\
\hline TransProb & $\begin{array}{l}34.3 \\
(0.88)\end{array}$ & $\begin{array}{l}42.7 \\
(0.83)\end{array}$ & $\begin{array}{l}38.0 \\
(0.87)\end{array}$ & $\begin{array}{l}52.8 \\
(1.22)\end{array}$ & $\begin{array}{l}71.1 \\
(1.00)\end{array}$ & $\begin{array}{l}60.6 \\
(1.15)\end{array}$ & $\begin{array}{l}24.3 \\
(0.55)\end{array}$ & $\begin{array}{l}39.7 \\
(1.1)\end{array}$ & $\begin{array}{l}30.1 \\
(0.70)\end{array}$ \\
\hline
\end{tabular}

Note that the PHOCUS results are from the " $3 \mathrm{~s}$ " implementation, which performed best on the corpus. Precision $(\mathrm{P})$, recall $(\mathrm{R})$, and F-score $(\mathrm{F})$ over word tokens $(\mathrm{T})$, word boundaries $(\mathrm{B})$, and lexicon items (L) resulting from the chosen word segmentation are shown. Standard deviations are shown in parentheses where available

find something unexpected: the DMCMC learner is out-performing the Ideal learner (DMCMC > Ideal: $p=.006)$. The Ideal learner is still out-performing the other two constrained learners, however (Ideal > DPM: $p=.031$, Ideal > DPS: $p<.001$ ).

Fourth, GGJ found that both their ideal learners tended to undersegment (putting multiple words together into one word), though the unigram learner did so more than the bigram learner (see Table 5 for examples).

One way to gauge whether undersegmentation is occurring is to look at the boundary precision and recall scores. When boundary precision is higher than boundary recall, undersegmentation is occurring; when the reverse is true, the model is oversegmenting (splitting single words into more than one word). If we examine Table 4, we can see that (as found by GGJ) the Ideal learners are undersegmenting, with the bigram model doing so less than the unigram model. Looking at our constrained learners, we can 
Table 5 GGJ ideal learner model performance: Unigram versus Bigram

Segmentations are shown in their English orthographic form, and undersegmentations are italicized

\begin{tabular}{ll}
\hline Unigram model & Bigram model \\
\hline youwant to see thebook & you want to see the book \\
look theres aboy & $\begin{array}{l}\text { look theres a boy } \\
\text { with his hat }\end{array}$ \\
and adoggie & and a doggie \\
you wantto lookatthis & you want to lookat this \\
lookatthis & lookat this \\
havea drink & have a drink \\
okay now & okay now \\
whatsthis & whats this \\
whatsthat & whats that \\
whatisit & whatis it \\
look canyou take itout & look canyou take it out \\
\hline
\end{tabular}

Fig. 3 Performance of unigram learners on whole utterances, first words, and last words

\section{Unigram learner performance: utterance portions}

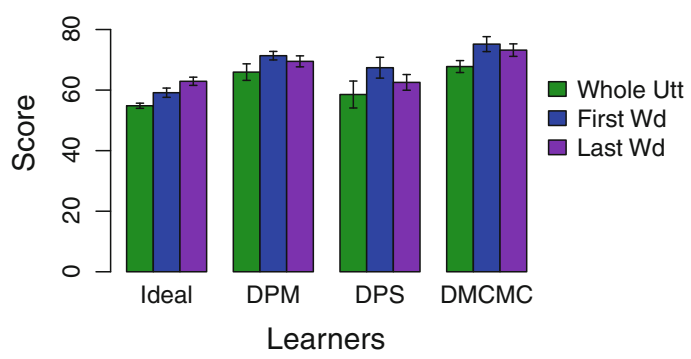

see that the unigram DMCMC learner is also undersegmenting. However, every other constrained model is oversegmenting, with the DPS learners being the most blatant oversegmenters; the bigram DMCMC learner appears to be oversegmenting the least.

We also examined performance on the first and last words in utterances, as compared to performance over the entire utterance, based on work by Seidl and Johnson (2006) who found that 7-month-olds are better at segmenting words that are either utteranceinitial or utterance-final [see Seidl and Johnson (2006) for detailed discussion on why this might be]. If our models are reasonable reflections of human behavior, we hope to find that their performance on the first and last words is better than their performance over the entire utterance. Moreover, they should perform equally on the first and last words in order to match infant behavior. Figures 3 and 4 show word token F-scores for unigram and bigram learners, respectively, for whole utterances, first words, and last words. Table 6 shows the significance test scores for comparing first word, last word, and whole utterance performance for each of the learners.

Looking first to the Bayesian unigram learners, we find that the DPM and DMCMC learners match infant behavior best by improving equally on first and last words, compared to whole utterances. The Ideal learner improves on both first and last words, 
Fig. 4 Performance of bigram learners on whole utterances, first words, and last words
Bigram learner performance: utterance portions

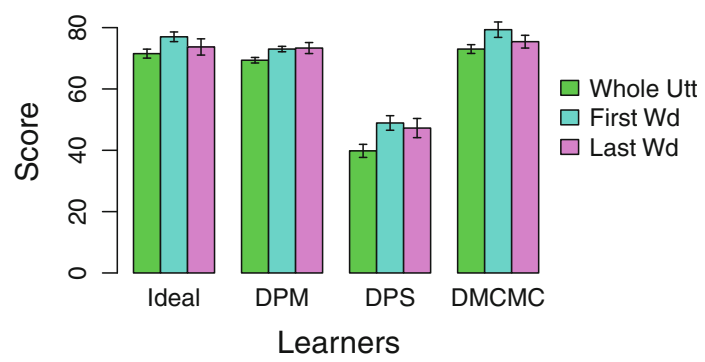

Table 6 Significance test scores (two tailed $t$-test) for comparisons between first word, last word, and whole utterance performance across the five test sets

\begin{tabular}{lccc}
\hline & First $\neq$ whole & Last $\neq$ whole & Last $\neq$ first \\
\hline Unigram models (words are not predictive) & & \\
GGJ-Ideal & .001 & $<.001$ & .003 \\
DPM & .008 & .046 & .108 \\
DPS & .008 & .130 & .038 \\
DMCMC & $<.001$ & .003 & .207 \\
Bigram models (words are predictive) & & \\
GGJ-Ideal & $<.001$ & .157 & .050 \\
DPM & $<.001$ & .005 & .730 \\
DPS & $<.001$ & .003 & .372 \\
DMCMC & .002 & .069 & .029 \\
\hline
\end{tabular}

Non-significant differences are italicized

but improves more for last words than for first words, making its performance slightly different than infants'. The DPS learner only achieves better performance for first words, making its performance even more different from infants'. Turning to the Bayesian bigram learners, we find that only the DPM and DPS learners are matching infants by improving equally on first and last word performance, compared to whole utterance performance. Both the Ideal and DMCMC learners only improve for first words, and not for last words.

\section{Discussion}

Through these simulations, we have made several interesting discoveries. First, though none of our constrained learners out-performed the best ideal learner (the bigram learner) on all measures, our constrained learners still were able to extract statistical information from the available data well enough to out-perform learners that segment by tracking transitional probability. Since transitional probability strategies have historically been strongly associated with the idea of "cognitively plausible statistical learning" in models of human language acquisition (e.g., Saffran et al. 1996; 
Table 7 Performance on test set 1 for DMCMC learners with varying samples per utterance

\begin{tabular}{lcccccccc}
\hline$s$ & 20000 & 10000 & 5000 & 2500 & 1000 & 500 & 250 & 100 \\
\hline \% Ideal learner samples & 11.0 & 5.7 & 2.8 & 1.4 & 0.57 & 0.28 & 0.14 & 0.057 \\
Unigram, $d=1$ & 69.8 & 68.5 & 65.5 & 63.5 & 63.4 & 60.0 & 56.9 & 51.1 \\
Bigram, $d=0.25$ & 74.9 & 71.8 & 68.3 & 66.1 & 64.6 & 61.2 & 59.9 & 60.9 \\
\hline
\end{tabular}

Learners were tested with the decay rate that yielded the best performance at 20000 samples per utterance (unigram $=1$, bigram $=0.25$ ). F-scores over word tokens are shown, as well as the processing comparison to the ideal learner (as measured by number of samples taken)

Saffran 2001; Perruchet and Desaulty 2008; Pelucchi et al. 2009), our result underscores how statistical learning can be considerably more successful than is sometimes thought when only transitional probability learners are considered. In addition, our online Bayesian learners also out-performed several recent statistical models of word segmentation with respect to identifying a reliable lexicon, while performing comparably at token and word boundary identification. Our results suggest that even with limitations on memory and processing, a learning strategy that focuses explicitly on identifying words in the input and optimizing a lexicon (as all our learners here do) may work better than one that focuses on identifying boundaries (as transitional probability learners and some recent statistical learning models do).

Second, we discovered that a bias that was helpful for the ideal learner-to assume words are predictive units-is not always helpful for constrained learners. This suggests that we must be careful in transferring the solutions we find for ideal learners to learners who have constraints on their memory and processing the way that humans do. In this case, we speculate that the reason some of our constrained learners do not benefit from the bigram assumption has to do with the algorithm's ability to search the hypothesis space; when tracking bigrams instead of just individual words, the learner's hypothesis space is much larger. It may be that some constrained learners do not have sufficient processing resources to find the optimal solution (and perhaps to recover from mistakes made early on). However, not all constrained learners suffer from this. There were constrained learners that benefited from the bigram assumption, which suggests less processing power may be required than previously thought to converge on good word segmentations. In particular, if we examine the DMCMC learner, we can decrease the number of samples per utterance to simulate a decrease in processing power. Table 7 shows the F-scores by word tokens for both the unigram and bigram DMCMC learner with varying samples per utterance. Though performance does degrade when processing power is more limited, these learners still out-perform the best phonemic transition probability learner variant we identified (which had scores around 38 for word tokens), even when sampling only $0.057 \%$ as much as the ideal learner. Moreover, the bigram assumption continues to be helpful, even with very little processing power available for the DMCMC learner.

If we constrain the ideal learner so it can only sample as often as the DMCMC learner does, we find that the unigram learner's segmentation performance is not quite as good as the DMCMC unigram learner's (see Table 8), though the bigram learner is much closer to (and in the case of the lexicon scores, better than) the DMCMC 
Table 8 Performance on test set 1 for DMCMC learners and ideal learners that only sample approximately as much as the DMCMC learners do

\begin{tabular}{|c|c|c|c|c|c|c|c|c|c|}
\hline & $\mathrm{TP}$ & TR & $\mathrm{TF}$ & $\mathrm{BP}$ & $\mathrm{BR}$ & $\mathrm{BF}$ & LP & LR & LF \\
\hline \multicolumn{10}{|c|}{ Unigram Learners (words are not predictive) } \\
\hline GGJ-Ideal & 62.7 & 49.6 & 55.4 & 90.5 & 63.5 & 74.7 & 55.8 & 73.7 & 63.5 \\
\hline DMCMC & 72.6 & 67.2 & 69.8 & 88.1 & 78.8 & 83.2 & 61.3 & 68.3 & 64.6 \\
\hline \multicolumn{10}{|c|}{ Bigram Learners (words are predictive) } \\
\hline GGJ-Ideal & 70.0 & 66.3 & 68.1 & 86.2 & 79.8 & 82.9 & 61.3 & 68.3 & 64.6 \\
\hline DMCMC & 68.6 & 72.3 & 70.4 & 81.2 & 87.4 & 84.2 & 59.5 & 60.5 & 59.9 \\
\hline
\end{tabular}

DMCMC learners sampled 20000 times per utterance with decay rate $=1$ for the Unigram learner and 0.25 for the Bigram learner. Ideal learners made 2000 iterations over the corpus, sampling every potential boundary once each iteration

Table 9 Posterior probability versus segmentation performance on test set 1 for Ideal and DMCMC learners

\begin{tabular}{lcccc}
\hline & Log posterior probability & TF & BF & LF \\
\hline Unigram learners (words are not predictive) & & & \\
GGJ-Ideal & -18077 & 55.4 & 74.7 & 63.5 \\
DMCMC & -19959 & 69.8 & 83.2 & 64.6 \\
Bigram Learners (words are predictive) & & & \\
GGJ-Ideal & -15642 & 68.1 & 82.9 & 64.6 \\
DMCMC & -16264 & 70.4 & 84.2 & 59.9 \\
\hline
\end{tabular}

Note that smaller absolute values of log posterior probability indicate segmentations that have higher probability under the model

bigram learner. One could imagine that the DMCMC learner scores so well because the DMCMC algorithm is simply more efficient than the Gibbs sampler used by the ideal learner-i.e., given the same number of total samples, DMCMC is able to find a higher probability segmentation than the Gibbs sampler. According to the results in Table 9, however, this is not the case: even when achieving higher segmentation scores, the DMCMC learner still finds a segmentation that actually has a lower posterior probability than its ideal learner counterpart. So it is not that the DMCMC learner is better at finding optimal solutions than the ideal learner-instead, it appears that some solutions that are sub-optimal with respect to posterior probability are actually better than those "optimal" solutions with respect to segmentation performance measures. This suggests that there could be something gained by the DMCMC learner's method of approximated inference if we are more interested in good segmentation performance (to be discussed further below).

Turning to the more general comparison of the ideal learner to the constrained learners, we made a surprising discovery-namely that some of our constrained unigram learners out-performed the ideal learner. This is somewhat counterintuitive, as one might naturally assume that less processing power would lead to equivalent if not worse performance. 
Table 10 Average performance of different learners on five test sets from the Pearl-Brent derived corpus

\begin{tabular}{|c|c|c|c|c|c|c|c|c|c|}
\hline & $\mathrm{TP}$ & TR & $\mathrm{TF}$ & $\mathrm{BP}$ & BR & $\mathrm{BF}$ & LP & LR & $\mathrm{LF}$ \\
\hline \multicolumn{10}{|c|}{ Unigram models (words are not predictive) } \\
\hline & 62.4 & 48.1 & 54.3 & 92.0 & 62.1 & 74.2 & 50.0 & 69.9 & 58.3 \\
\hline & $(0.52)$ & $(0.67)$ & $(0.62)$ & $(0.33)$ & $(0.53)$ & $(0.39)$ & $(0.76)$ & (1.10) & $(0.84)$ \\
\hline DPM & 53.6 & 66.1 & 59.2 & 66.7 & 88.5 & 76.1 & 60.9 & 38.5 & 47.2 \\
\hline & $(3.15)$ & (2.19) & (2.79) & $(2.37)$ & $(0.54)$ & (1.61) & (1.79) & $(1.70)$ & (1.81) \\
\hline DPS & 46.3 & 61.6 & 52.8 & 60.9 & 89.5 & 72.4 & 51.4 & 28.5 & 36.6 \\
\hline & $(5.48)$ & (3.66) & (4.87) & $(4.61)$ & (1.33) & $(3.10)$ & $(3.15)$ & $(4.22)$ & $(4.29)$ \\
\hline DMCMC & 67.5 & 61.0 & 64.1 & 86.3 & 74.5 & 79.9 & 53.8 & 61.0 & 57.2 \\
\hline & $(1.71)$ & (3.92) & $(2.80)$ & $(1.24)$ & $(4.08)$ & (1.96) & (3.11) & $(2.47)$ & $(2.82)$ \\
\hline \multicolumn{10}{|c|}{ Bigram models (words are predictive) } \\
\hline \multirow[t]{2}{*}{ GGJ-Ideal } & 70.4 & 68.3 & 69.4 & 85.6 & 82.0 & 83.7 & 60.5 & 65.5 & 62.9 \\
\hline & $(1.03)$ & $(0.75)$ & $(0.89)$ & $(0.78)$ & $(0.31)$ & $(0.53)$ & $(0.98)$ & $(0.67)$ & $(0.80)$ \\
\hline \multirow[t]{2}{*}{ DPM } & 61.9 & 70.3 & 65.9 & 75.2 & 89.6 & 81.7 & 61.0 & 48.9 & 54.3 \\
\hline & $(1.58)$ & $(0.97)$ & $(1.27)$ & $(1.16)$ & $(0.83)$ & $(0.61)$ & $(0.79)$ & $(1.01)$ & $(0.68)$ \\
\hline \multirow[t]{2}{*}{ DPS } & 32.3 & 48.4 & 38.7 & 52.8 & 90.5 & 66.6 & 37.6 & 23.7 & 29.1 \\
\hline & $(4.99)$ & $(4.73)$ & $(5.10)$ & $(3.47)$ & $(0.95)$ & $(2.63)$ & $(1.62)$ & $(1.69)$ & (1.74) \\
\hline \multirow[t]{2}{*}{ DMCMC } & 69.2 & 73.1 & 71.1 & 81.1 & 87.6 & 84.2 & 52.7 & 53.0 & 52.8 \\
\hline & (1.19) & $(0.96)$ & (1.08) & $(0.89)$ & $(0.49)$ & $(0.69)$ & $(1.41)$ & $(1.37)$ & (1.34) \\
\hline
\end{tabular}

Precision (P), recall (R), and F-score (F) over word tokens (T), word boundaries (B), and lexicon items (L) resulting from the chosen word segmentation are shown. Standard deviations are shown in parentheses

To rule out the possibility that these results are an artifact of this particular corpus, we tested our learners on a larger corpus of English, the Pearl-Brent derived corpus available through CHILDES (MacWhinney 2000). This corpus contains child-directed speech to children between 8 and 9 months old, consisting of 28,391 utterances $(96,920$ word tokens, 3,213 word types, average words per utterance: 3.4 , average phonemes per word: 3.6). In Table 10, we report the learners' performance on five test sets generated from this corpus (these were generated the same way as the ones from the Bernstein-Ratner corpus were). The same surprising performance trend appears, where the DMCMC unigram learner is out-performing the Ideal unigram learnerthough only with respect to tokens and word boundaries, and not with respect to lexicon items.

We subsequently looked at the errors being made by both the ideal and the DMCMC unigram learners on these English corpora, and discovered a potential cause for the surprising behavior. It turns out that the ideal learner makes many more undersegmentation errors on highly frequent bigrams consisting of short words (e.g., can you, do you, and it's a segmented as canyou, doyou, and itsa) while the DMCMC learner does not undersegment these bigrams. When the DMCMC learner does make errors on frequent items that are different from the errors the ideal learner makes, it tends to oversegment, often splitting off sequences that look like English derivational morphology, such as "-s" (plural or 3rd sg present tense) and "-ing” (progressive) 
Table 11 Analysis of unshared errors made by the ideal and DMCMC unigram learners for items occurring 7 or more times in the first test set of each corpus

\begin{tabular}{lcc}
\hline Corpus & Ideal learner (undersegmentation) & DMCMC learner (oversegmentation) \\
\hline Bernstein-Ratner & 749 & 62 \\
Pearl-Brent & 1671 & 185 \\
\hline
\end{tabular}

(e.g., ringing segmented as ring ing, and flowers segmented as flower s). If we survey the errors made by each learner for items occurring 7 or more times in the first test set of each English corpus and which are not shared (i.e., only one learner made the error), we find the DMCMC learner's additional errors are far fewer than the ideal learner's additional errors (Table 11).

Why might this particular error pattern occur? A possible explanation for this error pattern is related to the ideal learner's increased processing capabilities. Specifically, the ideal learner is granted the memory capacity to survey the entire corpus for frequency information and update its segmentation hypotheses for utterances occurring early in the corpus at any point during learning. This allows the ideal unigram learner to notice that certain short items (e.g., actual words like it's and $a$ ) appear very frequently together. Given that it cannot represent this mutual occurrence any other way, it will decide to make these items a single lexical item; moreover, it can fix its previous "errors" that it made earlier during learning when it thought these were two separate lexical items. In contrast, the DMCMC learner does not have this omniscience about item frequency in the corpus, nor as much ability to fix "errors" made earlier in the learning process. This results in the DMCMC learner leaving these short items as separate, particularly when encountered in earlier utterances. As they then continue to exist in the lexicon as separate lexical items, undersegmentation errors do not occur nearly as much.

In summary, more processing power and memory capacity does appear to hurt the inference process of the ideal unigram learner, even if that learner identifies a segmentation with a higher posterior probability. This behavior is similar to Newport's (1990) "Less is More" hypothesis for human language acquisition, which proposes that limited processing abilities are advantageous for tasks like language acquisition because they selectively focus the learner's attention. With this selective focus, children are better able to home in on the correct components for language since they do not consider as much complex information. Transferring this idea to our unigram learners, the more limited inference process of the DMCMC learner focuses its attention only on the current frequency information and does not allow it to view the frequency of the corpus as a whole. Coupled with this learner's more limited ability to correct its initial hypotheses about lexicon items, this leads to superior segmentation performance. We note, however, that this superior performance is mainly due to the unigram learner's inability to capture word sequence predictiveness; when it sees items appearing together, it has no way to capture this behavior except by assuming these items are actually one word. Thus, the ideal unigram learner's additional knowledge causes it to commit more undersegmentation errors in its quest to find the segmentation with the highest posterior probability. The bigram learner, on the other hand, does not have 
this problem — and indeed we do not see the DMCMC bigram learner out-performing the ideal bigram learner.

Turning to general undersegmentation behavior, we also discovered that the tendency to undersegment the corpus depends on how constraints are implemented in our learners, as well as whether the learners assume words are predictive or not. According to Peters (1983), English children tend to make errors that indicate undersegmentation rather than oversegmentation, so perhaps learners that undersegment are a better match for children's behavior. Here, the Bayesian learners that undersegmented on the English data were both of the ideal learners as well as the unigram DMCMC learner.

Another finding is that models differ on their ability to match infant word segmentation behavior at utterance edges (the first word and the last word). Some of our constrained models are in fact better able to match infant behavior on this measure than our ideal models. Seidl and Johnson (2006) review a number of proposed explanations of why utterance edges are easier, including perceptual/prosodic salience, cognitive biases to attend more to edges (including recency effects), or the pauses at utterance boundaries. In our results, we find that all of the models find utterance-initial words easier to segment, and most of them also find utterance-final words easier. Since none of the algorithms include models of perceptual salience, our results suggest that this explanation is probably unnecessary to account for the edge effect, especially for utterance-initial words. Rather, it seems simpler to assume that the pauses at utterance boundaries make segmentation easier by eliminating the ambiguity of one of the two boundaries of the word.

However, if this were the only effect at utterance edges, then we would expect all of our models to find both initial and final words easier. In fact, some of them, including the ideal bigram learner, find only initial words easier. This finding suggests that some other statistical property of final words actually make them more difficult than initial words for (at least some) purely statistical learners. For example, the words and phrases that end sentences (often nouns or verbs) may be more variable or infrequent than the words that start sentences (often pronouns or determiners). Since utterance-final words seem to be at least as easy for infants as utterance-initial words, a recency effect could be playing an important role here. However, in light of the varying results of the different models, further analysis of the statistical properties of utterance-final versus utterance-initial words is warranted before drawing any strong conclusions.

\section{Conclusions and Future Work}

One moral of this investigation is that a simple intuition about human cognition, such as having memory and processing limitations, can be cashed out multiple ways in online learning algorithms. Here, we examined limitations such as processing utterances incrementally and implementing recency effects with exponential decay functions. Having explored several learner instantiations incorporating this intuition, we find that the learning assumptions or biases that work best depend on how limitations are implemented. And in fact, some biases that are helpful for an ideal learner, 
such as using context to guide hypotheses, may hinder a constrained learner with more limited memory and processing resources. On a related note, if the learner does not use word context, having less memory and processing resources may in fact be beneficial.

We view these investigations as a first step towards understanding how to translate computational-level solutions into algorithmic-level ones for language acquisition, as there are clearly other ways of implementing constrained algorithms. One question we might ask is whether any given learner represented by a combination of a model and a constrained learning algorithm (such as our constrained learners here) can be represented solely by a model that explicitly defines those same constraints. Then, if this second model could be optimized, we would find it yields the same answer as the constrained learners here. This would place what we are currently considering algorithmic-level constraints back into the computational-level definition of the model. While this is possible, it is by no means certain and it could very well be that this latter kind of model is considerably more complicated.

It is also useful to ask if the effects discovered here are robust, and persist across different languages. If the learning model presented here is meant to be a first pass language-independent method for word segmentation, we would want it to be successful in languages besides English. Moreover, with respect to empirical grounding, we can take further inspiration from what is known about the representations infants attend to, and allow our algorithms to have knowledge of syllables (Jusczyk et al. 1999a), to track stressed and unstressed phonemes/syllables separately (Curtin et al. 2005; Pelucchi et al. 2009), and to have additional prior phonotactic knowledge argued to be universal in human language (e.g., Blanchard et al. 2010).

The transition from a computational-level solution for an acquisition problem to the algorithmic-level approximation may not necessarily be straightforward. By integrating what we know of the human ability to utilize available statistical information with what we know of human limitations, we can come to understand how infants accomplish the things they do.

Acknowledgments We would like to thank the audiences at the PsychoComputational Models of Human Language workshop in 2009, BUCLD 34, three anonymous reviewers, Alexander Clark, William Sakas, Tom Griffiths, and Michael Frank. We would also like to give a special thanks to Jim White for his insight about the differences in performance between the ideal and online Bayesian learners. This work was supported by NSF grant BCS-0843896 to the first author and CORCL grant MI 14B-2009-2010 to the first and third authors.

Open Access This article is distributed under the terms of the Creative Commons Attribution Noncommercial License which permits any noncommercial use, distribution, and reproduction in any medium, provided the original author(s) and source are credited.

\section{Appendix: Phoneme Encoding of Corpus}

See Fig. A1. 


\begin{tabular}{|c|l|c|l|}
\hline \multicolumn{5}{|c|}{ Consonants } \\
\hline \hline ASCII & Ex. & ASCII & Ex. \\
\hline \hline D & THe & h & Hat \\
G & Jump & k & Cut \\
L & bottLe & I & Lamp \\
M & rhythM & m & Man \\
N & siNG & n & Net \\
S & SHip & p & Pipe \\
T & THin & r & Run \\
W & WHen & s & Sit \\
Z & aZure & t & Toy \\
b & Boy & v & View \\
c & CHip & w & We \\
d & Dog & y & You \\
f & Fox & z & Zip \\
g & Go & $\sim$ & buttON \\
\hline
\end{tabular}

\begin{tabular}{|c|l|}
\multicolumn{2}{|c|}{ Vowels } \\
\hline ASCII & Ex. \\
\hline \hline$\&$ & thAt \\
6 & About \\
7 & bOY \\
9 & flY \\
A & bUt \\
E & bEt \\
I & bIt \\
0 & 1 AW \\
$\mathrm{Q}$ & bOUt \\
U & pUt \\
a & hOt \\
$\mathrm{e}$ & bAY \\
$i$ & bEE \\
o & bOAt \\
u & bOOt \\
\hline
\end{tabular}

Rhotic Vowels
\begin{tabular}{|c|l|}
\hline ASCII & Ex. \\
\hline \hline$\#$ & ARe \\
$\%$ & fOR \\
( & hERE \\
) & lURE \\
$*$ & hAIR \\
3 & bIRd \\
R & buttER \\
\hline
\end{tabular}

Fig. A1 Phoneme encoding. Taken with permission from Goldwater et al. (2007)

\section{References}

Anderson, J. R., \& Schooler, L. J. (2000). The adaptive nature of memory. In E. Tulving \& F. I. M. Craik (Eds.), The Oxford handbook of memory (pp. 557-570). Oxford: Oxford University Press.

Bernstein-Ratner, N. (1984). Patterns of vowel modification in motherese. Journal of Child Language, 11, 557-578.

Blanchard, D., Heinz, J., \& Golinkoff, R. (2010). Modeling the contribution of phonotactic cues to word segmentation. Journal of Child Language, 27, 487-511.

Brent, M. (1999). An efficient, probabilistically sound algorithm for segmentation and word discovery. Machine Learning, 34, 71-105.

Brown, S., \& Steyvers, M. (2009). Detecting and predicting changes. Cognitive Psychology, 58, 49-67.

Christiansen, M., Allen, J., \& Seidenberg, M. (1998). Learning to segment speech using multiple cues: A connectionist model. Language and Cognitive Processes, 13, 221-268.

Curtin, S., Mintz, T., \& Christansen, M. (2005). Stress changes the representational landscape: Evidence from word segmentation in infants. Cognition, 96, 233-262.

Ferguson, T. (1973). A Bayesian analysis of some nonparametric problems. Annals of Statistics, 1, 209-230.

Fleck, M. (2008). Lexicalized phonotactic word segmentation. In Proceedings of the association for computational linguistics (pp. 130-138).

Frank, M. C., Goodman, N. D., \& Tenenbaum, J. (2009). Using speakers' referential intentions to model early cross-situational word learning. Psychological Science, 20, 579-585.

Gambell, T., \& Yang, C. (2006). Word segmentation: Quick but not dirty. Manuscript. New Haven: Yale University.

Griffiths, T. L., Chater, N., Kemp, C., Perfors, A., \& Tenenbaum, J. B. (2010). Probabilistic models of cognition: Exploring representations and inductive biases. Trends in Cognitive Sciences, 14, 357-364.

Griffiths, T. L., Kemp, C., \& Tenenbaum, J. B. (2008). Bayesian models of cognition. In Ron Sun (Ed.), The Cambridge handbook of computational cognitive modeling. Cambridge: Cambridge University Press.

Griffiths, T. L., \& Tenenbaum, J. B. (2005). Structure and strength in causal induction. Cognitive Psychology, 51, 354-384.

Goldwater (2006). Nonparametric Bayesian models of lexical acquisition. Ph.D. thesis, Brown University.

Goldwater, S., Griffiths, T., \& Johnson, M. (2007). Distributional cues to word boundaries: Context is important. In H. Caunt-Nulton, S. Kulatilake, \& I. Woo (Eds.), BUCLD 31: Proceedings of the 31st annual Boston university conference on language development (pp. 239-250). Somerville, MA: Cascadilla Press.

Goldwater, S., Griffiths, T. L., \& Johnson, M. (2009). A Bayesian framework for word segmentation: Exploring the effects of context. Cognition, 112(1), 21-54. 
Hewlett, D., \& Cohen, P. (2009). Bootstrap voting experts. In Proceedings of the twenty-first international joint conference on artificial intelligence (IJCAI-09) (pp. 1071-1076). Available at http://www. ijcai.org/papers09/contents.php.

Johnson, E., \& Jusczyk, P. (2001). Word segmentation by 8-month-olds: When speech cues count more than statistics. Journal of Memory and Language, 44, 548-567.

Johnson, M., Griffiths, T., \& Goldwater, S. (2007). Bayesian inference for PCFGs via Markov Cain Monte Carlo. In Proceedings of the meeting of the North American association for computational linguistics.

Jusczyk, P., Goodman, M., \& Baumann, A. (1999a). Nine-month-olds' attention to sound similarities in syllables. Journal of Memory \& Language, 40, 62-82.

Jusczyk, P., Hohne, E., \& Baumann, A. (1999b). Infants' sensitivity to allophonic cues for word segmentation. Perception and Psychophysics, 61, 1465-1476.

Juszcyk, P., Houston, D., \& Newsome, M. (1999c). The beginnings of word segmentation in Englishlearning infants. Cognitive Psychology, 39, 159-207.

MacWhinney, B. (2000). The CHILDES project: Tools for analyzing talk. Mahwah, NJ: Lawrence Erlbaum Associates.

Marr, D. (1982). Vision. San Francisco: Freeman.

Marthi, B., Pasula, H., Russell, S., \& Peres, Y., et al. (2002). Decayed MCMC Filtering. In Proceedings of 18th UAI (pp. 319-326).

Mattys, S., Jusczyk, P., Luce, P., \& Morgan, J. (1999). Phonotactic and prosodic effects on word segmentation in infants. Cognitive Psychology, 38, 465-494.

McClelland, J. L., Botvinick, M. M., Noelle, D. C., Plaut, D. C., Rogers, T. T., Seidenberg, M. S., \& Smith, L. B. (2010). Letting structure emerge: Connectionist and dynamical systems approaches to understanding cognition. Trends in Cognitive Sciences, 14, 348-356.

Morgan, J., Bonamo, K., \& Travis, L. (1995). Negative evidence on negative evidence. Developmental Psychology, 31, 180-197.

Newport, E. (1990). Maturational constraints on language learning. Cognitive Science, 14, 11-28.

Oaksford, M., \& Chater, N. (1998). Rational models of cognition. Oxford, England: Oxford University Press.

Pelucchi, B., Hay, J., \& Saffran, J. (2009). Learning in reverse: Eight-month-old infants track backward transitional probabilities. Cognition, 113, 244-247.

Perruchet, P., \& Desaulty, S. (2008). A role for backward transitional probabilities in word segmentation? Memory and Cognition, 36, 1299-1305.

Peters, A. (1983). The Units of Language Acquisition, Monographs in Applied Psycholinguistics. New York: Cambridge University Press.

Saffran, J., Aslin, R., \& Newport, E. (1996). Statistical learning by 8-month-olds. Science, 274, 1926-1928.

Saffran, J. R. (2001). The use of predictive dependencies in language learning. Journal of Memory and Language, 44, 493-513.

Sanborn, A. N., Griffiths, T. L., \& Navarro, D. J. (in press). Rational approximations to rational models: Alternative algorithms for category learning. Psychological Review.

Seidl, A., \& Johnson, E. (2006). Infant word segmentation revisited: Edge alignment facilitates target extraction. Developmental Science, 9(6), 565-573.

Shi, L., Griffiths, T. L., Feldman, N. H., \& Sanborn, A. N. (in press). Exemplar models as a mechanism for performing Bayesian inference. Psychonomic Bulletin \& Review.

Swingley, D. (2005). Statistical clustering and contents of the infant vocabulary. Cognitive Psychology, 50, 86-132.

Teh, Y., Jordan, M., Beal, M., \& Blei, D. (2006). Hierarchical Dirichlet processes. Journal of the American Statistical Association, 101(476), 1566-1581.

Tenenbaum, J., \& Griffiths, T. (2001). Generalization, similarity, and Bayesian inference. Behavioral and Brain Sciences, 24, 629-641.

Tenenbaum, J., Griffiths, T., \& Kemp, C. (2006). Theory-based models of inductive learning and reasoning. Trends in Cognitive Sciences, 10, 309-318.

Thiessen, E., \& Saffran, J. R. (2003). When cues collide: Use of stress and statistical cues to word boundaries by 7- to 9-month-old infants. Developmental Psychology, 39, 706-716.

Xu, F., \& Tenenbaum, J. B. (2007). Word learning as Bayesian inference. Psychological Review, 114, 245272. 